\title{
CAM3 bias over the Arctic region during northern winter studied with a linear stationary model
}

\author{
Richard Grotjahn • Lin-Lin Pan · Joseph Tribbia
}

Received: 6 January 2010/ Accepted: 12 February 2011/Published online: 6 March 2011

(c) The Author(s) 2011. This article is published with open access at Springerlink.com

\begin{abstract}
This study builds upon two prior papers, which examine Arctic region bias of CAM3 (NCAR Community Atmosphere Model version 3) simulations during winter. CAM3 output is compared with ECMWF (European Centre for Medium-Range Weather Forecasts) 40 year reanalysis (ERA-40) data. Our prior papers considered the temperature and the vorticity equation terms and demonstrated that diabatic, transient, and linear terms dominate nonlinear bias terms over most areas of interest. Accordingly, this paper uses a linearized form of the model's dynamical core equations to study aspects of the forcing that lead to the CAM3 biases. We treat the model's long term winter bias as a solution to a linear stationary wave model (LSWM). Key features of the bias in the vorticity, temperature, and $\ln$ of surface pressure $(=q)$ fields are shown at medium resolution. The important features found at medium resolution are captured at the much lower LSWM resolution. The Arctic q bias has two key features: excess q over the Barents Sea and a missing Beaufort High (negative maximum q bias) to the north of Alaska and eastern Siberia. The forcing fields are calculated by the LSWM. Horizontal advection tends to create multi-polar combinations of negative and positive extrema in the forcing. The positive and negative areas of forcing approximately match corresponding areas in the bias. There is a broad relation between cold bias with elevated $\mathrm{q}$ bias, as expected from classical theory. Forcing in related
\end{abstract}

R. Grotjahn $(\square)$

Department of Land, Air and Water Resources,

University of California, Davis, CA 95616, USA

e-mail: grotjahn@ucdavis.edu

L.-L. Pan · J. Tribbia

National Center for Atmospheric Research, Boulder, CO, USA quantities: near surface vorticity and surface pressure combine to produce the sea level pressure bias.

Keywords CAM3 - Arctic simulation bias - Northern hemisphere storm tracks $\cdot$ Linear stationary wave model

\section{Introduction}

This study examines the Northern Hemisphere winter bias of the NCAR Community Atmosphere Model version 3 (CAM3). CAM3 is described by Collins et al. (2004). The Arctic surface climate has particular focus and that has been studied by DeWeaver and Bitz (2006) as well.

Previously we showed (Pan et al. 2009, hereafter PGT; Grotjahn et al. 2011, hereafter GPT) that the CAM3 storm tracks have different dynamics than those in ERA-40. In PGT and GPT, as here, Northern Hemisphere winter (December-February) is studied. We emphasized the North Atlantic storm track (NAST) because of its very large location error on the downstream end and the impact that error has on Arctic surface climate. By the Greenwich meridian, the NAST in CAM3 data is about $10^{\circ}$ latitude south of the NAST in ERA-40 data. In PGT the CAM3 meridional heat fluxes (band passed 2-8 days frequency) were less than in ERA-40 data. However, the precipitation was much greater in CAM3's NAST than in ERA-40. In GPT the band passed winds within the NAST were found to be much weaker, such that KE was about $3 / 4$ of the ERA-40 value and enstrophy was only a third of the ERA40 value. While some of the enstrophy and KE error could be attributed to the higher resolution used in creating the ERA-40 data (before being spectrally truncated and then regridded to match the T42 CAM3 data in PGT and GPT) in fact, essentially all wavelengths, including planetary 
waves, have less amplitude in CAM3. GPT also noted that the CAM3 surface bias on the European side of the Arctic was due to a storm track related quantity (the divergence term in the vorticity tendency equation) and the local bias in the divergence term reinforced a PGT result that linked higher precipitation over England with higher pressure where CAM3 has a relative high pressure bias (near Novaya Zemlya, Barents Sea, and adjacent Russian coast).

CAM3 has another long standing difficulty in reproducing the Beaufort high. GPT showed how the anticyclonic vorticity of the Beaufort high in ERA-40 data was advected from the central Siberian coast first towards the north pole, then zonally (eastward) around the Arctic. This advection was largely missing from CAM3 data and what little occurred was being cancelled by the divergence term that locally generated cyclonic vorticity (the local divergence term is negligible in ERA-40).

A piece of the puzzle missing in the prior studies is how much of these Arctic region sea level pressure (SLP) biases can be related to local factors and how much to remote causes. A linear stationary wave model (LSWM) can provide some insight into the influence of remote and local forcing on the time mean, but it cannot identify travelling effects (such as a storm track error that originates upstream) except for their local time mean consequence or if such travelling effects create a time mean wavetrain response from the remote region to the region of interest. A LSWM requires an assumption that the bias is primarily driven by processes that are linear in the bias. To evaluate that assumption, PGT and GPT demonstrated that nonlinear terms (quadratic in the bias) are negligible relative to linear combinations of the bias and the other forcing terms (transient contributions to the time mean and diabatic processes). With the exceptions of the Iberian Peninsula and regions of large topographic gradients (like Greenland), the nonlinear terms were found to be much smaller than the transient and diabatic terms of the vorticity and temperature equations. So, this third paper in the sequence examines the bias as a forced stationary response.

\section{Linear model}

The LSWM is a linear operator on the bias. The LSWM linear operator is similar to the model described in Branstator (1990). However, the temperature part of the operator is the temperature bias equation studied by PGT. The vorticity part of the linear operator is the vorticity bias equation studied by GPT. The equations for vorticity, divergence, temperature, and ln of surface pressure (q) are formed by evaluating the time mean equation for each variable using CAM3 data then subtracting the same terms evaluated using observation-based data. The CAM3 data
Fig. 1 Northern hemisphere bias of a T42, 29level historical climate simulation (1979-1998) by CAM3. Shown are vorticity (a, d; top row), divergence (b, e; second row), and temperature (c, $\mathbf{f} ;$ third row) at three levels: $\sigma=0.1$ (a-c; left column), $\sigma=0.5(\mathbf{d}-\mathbf{f} ;$ right column). $\mathbf{j}$ The contour interval varies between variables, but is the same for all plots of a specific variable. On next page are shown vorticity (g), divergence (h), and temperature (i) at level: $\sigma=0.95$. Also shown is the $\mathrm{q}=\ln \left(\mathrm{P}_{\mathrm{s}}\right)$ bias $(\mathbf{j})$ and the bias in sea level pressure (SLP) in (k). The contour interval varies between variables, but is the same for all plots of a specific variable

used here are obtained by running a 20 year AMIP (Atmospheric Model Intercomparison Project) type simulation using historical climate boundary specifications from 1979 to 1998 . The model version used has 26 levels in the vertical and the horizontal resolution is triangular truncation at wavenumber 42 (T42). The output is saved 4 times daily. Only the Northern Hemisphere winter months: December, January, and February are studied. The observational data used for comparison here are gridded $4 \times$ daily ERA-40 reanalysis data (Uppala et al. 2005) from 1979 to 1998 . The variables used include temperature, zonal wind, meridional wind, and vertical velocity in p-coordinates.

The LSWM uses the same equations as Branstator (1990) with a numerical resolution in the horizontal of R12 (rhomboidal truncation at zonal wavenumber 12) and ten vertical levels (equally-spaced in a terrain-following coordinate, $\sigma=1-\mathrm{P} / \mathrm{P}_{\mathrm{S}}$ where $\mathrm{P}_{\mathrm{S}}$ is the pressure at the Earth's surface). Rhomboidal truncation is used due to its higher resolution in the polar regions (compared with tropical areas).

The model is based on the same formulation of the dynamical core within CAM3. One difference is that a larger damping rate is used to obtain linear stationary solutions following a practice often used in linear models (e.g. Pan et al. 2006). The larger damping could affect the growth rate if we were examining that property (but we are not). Instead, the structure of the solution we focus upon is little changed (for example) by reducing the damping by a factor of 10 .

Another difference from the CAM3 dynamical core is this model works with the 'bias' forms of the equations (see PGT and GPT for details). Each bias equation is constructed by the following steps: (1) apply a time mean to each equation and separate contributions from the time mean and transient variables, (2) perform step 1 for ERA40 data and for CAM3 data, then (3) subtract the equation based on ERA-40 values from the corresponding equation using CAM3 values to get the 'bias' form of that equation. Since the bias is a difference between the time means for CAM3 and ERA-40, the terms in the bias form of the equation include one or more of: ERA-40 time mean, the bias, and transients (to the extent they contribute to the 
a

$$
\sigma=0.1
$$

VOR BIAS

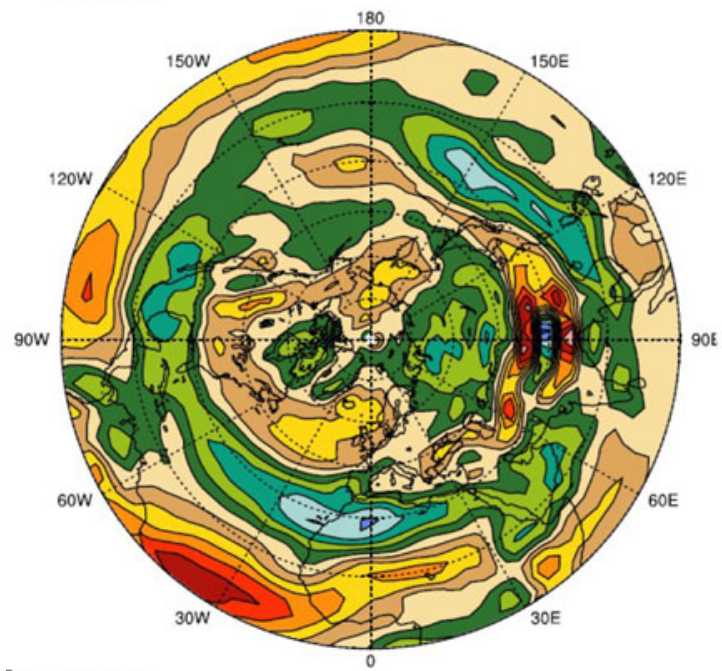

b DIV BIAS

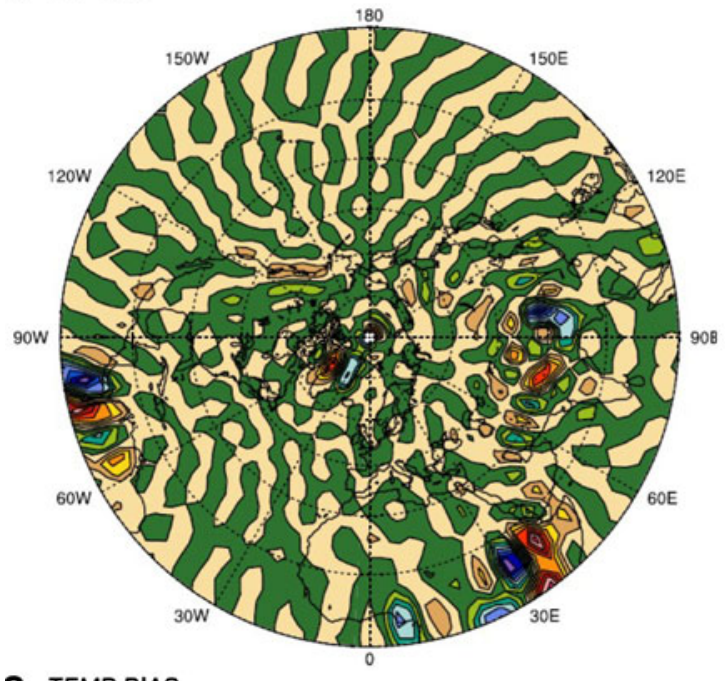

C TEMP BIAS

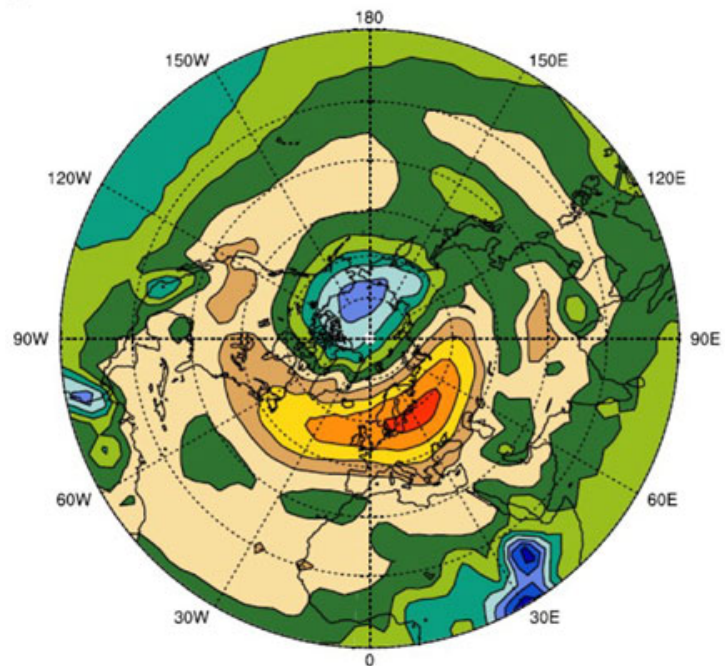

d

$$
\sigma=0.5
$$

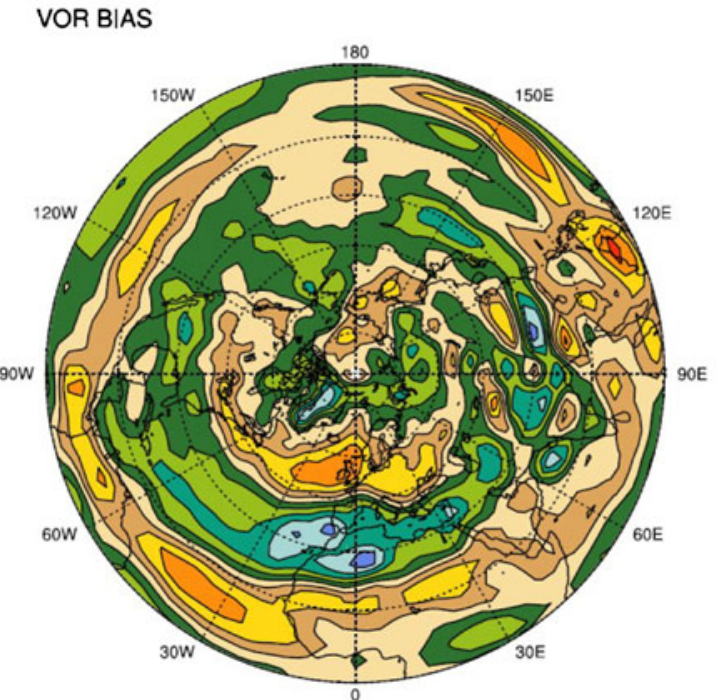

e DIV BIAS

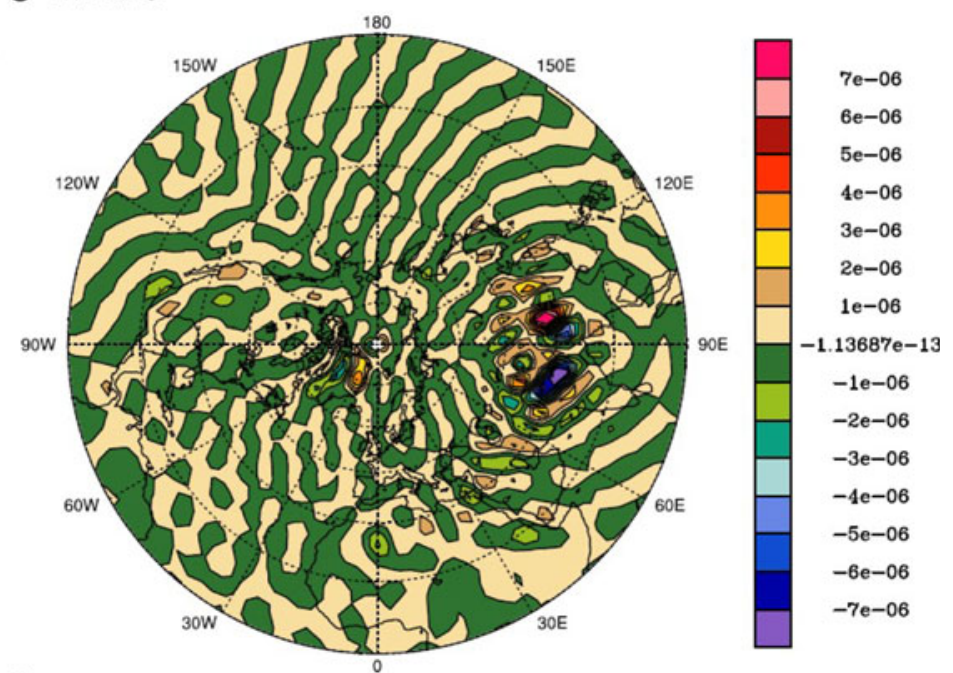

f TEMP BIAS

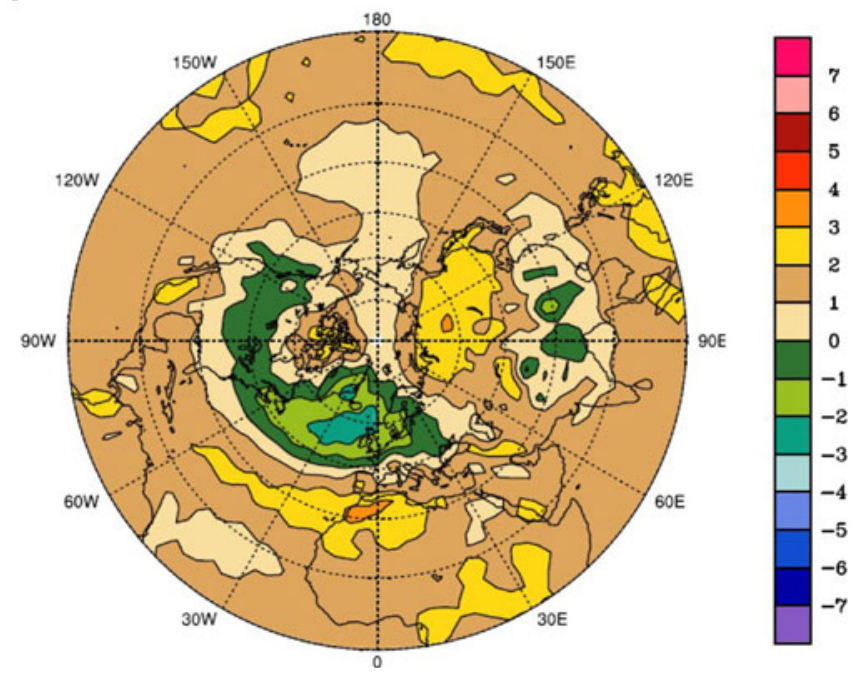




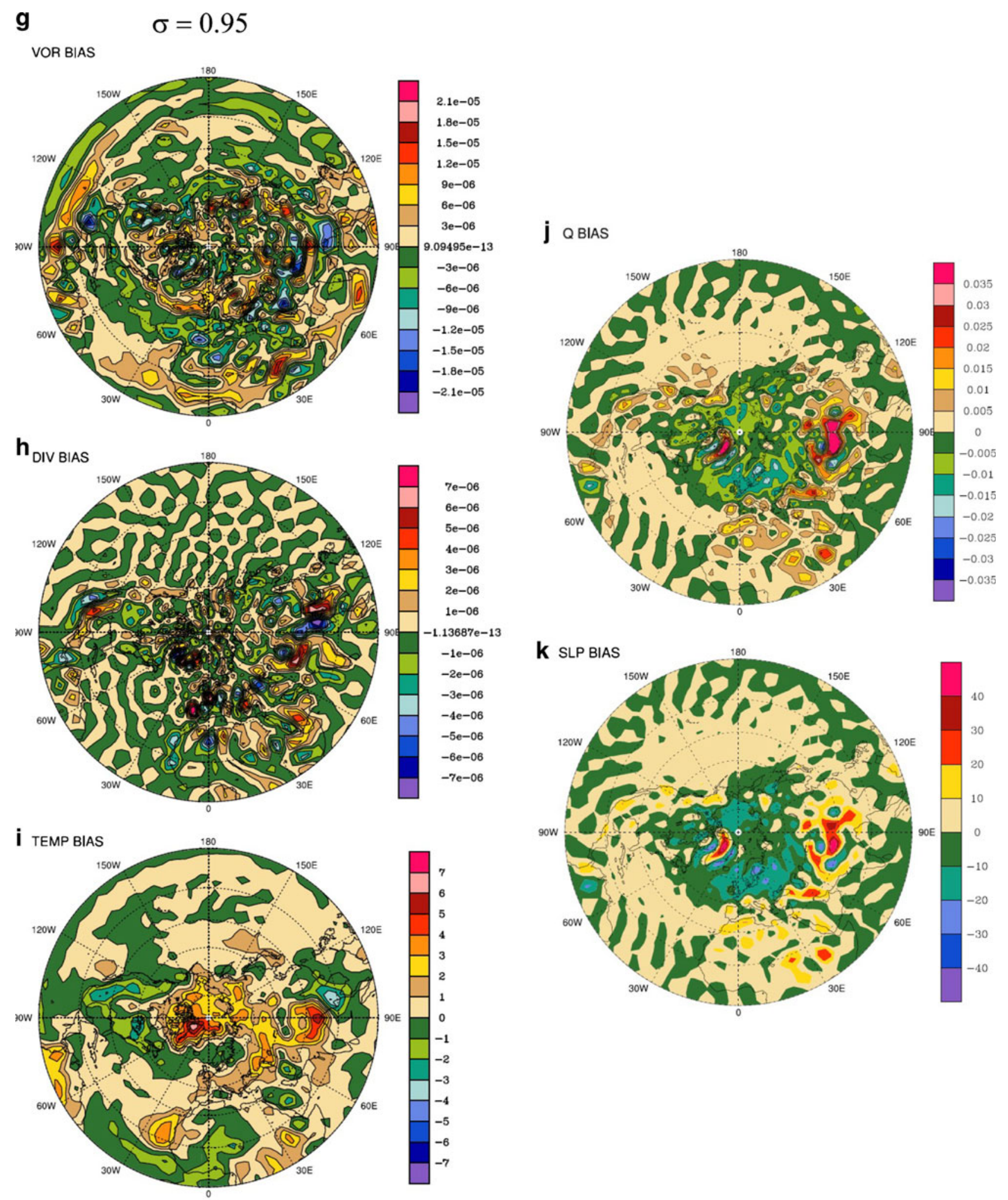

Fig. 1 continued 
time mean). The terms in each resultant bias equation that are a linear function of the bias are collected to define the linear operator ' $\mathrm{L}$ ' all other terms (including all the nonlinear contributions) are collected into a forcing term ' $\mathrm{F}$ '.

The LSWM can thereby be written as a matrix equation of the form:

$\mathrm{LX} \overline{\mathrm{X}}_{\mathrm{a}}=\mathrm{F}$

In (1) $\bar{X}_{a}$ is a vector containing all the spherical harmonics at all the vertical levels for CAM3 bias in the four model variables: vorticity $(\zeta)$, divergence (D), temperature $(\mathrm{T})$, and natural logarithm of surface pressure $\left(\mathrm{q}=\ln \left(\mathrm{P}_{\mathrm{S}}\right)\right)$. The bias is defined as the seasonal time mean model value minus the corresponding seasonal time mean value from the ERA-40 reanalysis dataset. In (1) L is a very large square matrix linear operator upon the bias. It includes all terms that are a linear function of the bias. Hence, L includes every term, and only those terms, in which the bias appears once. $\mathrm{L}$ excludes any term where the bias multiplies any other bias, such as the bias in another variable; such terms are referred to here as 'nonlinear bias terms' and they are not kept in the model equations. The nonlinear bias terms were examined for the temperature equation by PGT and for the vorticity equation by GPT and in both cases the nonlinear bias terms were much smaller than linear, transient, and diabatic terms. On the strength of those analyses in PGT and GPT we ignore the nonlinear bias terms here. In (1) F includes all forcing terms needed to arrive at the model bias when that bias is operated upon by L. Physically, F includes diabatic processes and transient contributions to the time mean in the model equations.

We do not parse the forcing further, neither into various transients nor parts of the diabatic processes for several reasons. One reason is that it is unclear where to divide the forcing to isolate specific physical processes. Such a division is beyond the limited scope of this study. However, PGT and GPT already have extensive discussions of the bias in various categories: diabatic heating (precipitation, surface sensible heat flux, net radiation), transients, and nonlinear (in the bias) sources. The reader is referred to those papers.

The procedure for using (1) is as follows. The CAM3 biases in $\zeta, \mathrm{D}, \mathrm{T}$, and $\mathrm{q}$ are obtained on pressure coordinates. These data are regridded to the low resolution of the LSWM and to the model's terrain following coordinate using NCAR Command Language (NCL) routines. Performing the operation on the left hand side of (1) (i.e.L $\bar{X}_{a}$ ) obtains the forcing vector $F$. One can then reverse the operation to obtain the original input bias field from the forcing vector. Clearly, the interesting experiments are to use portions of the bias to obtain the forcing and portions of the forcing to obtain parts of the total bias.
Finding the forcing from the bias is a simple matrixvector multiplication and that is the subject of the next section. In Sect. 4, the forcing is separately used in certain regions of the globe and the bias obtained in two regions of interest in the Arctic. That analysis procedure estimates what portion of the Arctic bias is being generated by local versus remote forcing.

\section{Bias and LSWM forcing fields}

The truncation from T42 to lower resolution (R12) simplifies the structure of the bias field. Figure 1 shows CAM3 bias of the LSWM fields at several representative levels using T42 data. Figure 2 shows corresponding fields but at the lower truncation (R12) of the LSWM. The biases in vorticity and temperature are shown in Figs. 1 and 2 at levels intended to be representative of the stratosphere and middle and lower troposphere. In the latitudes higher than $30 \mathrm{~N}$ most of the broad features present at T42 resolution are also present at R12, though smoother; some features have $2 / 3$ the magnitude of the higher resolution value.

Model bias in the Arctic surface climate is a focus of this research. The SLP bias (inferred from the $\mathrm{q}$ bias and also shown in Figs. 1k, 2k) has all the primary features at R12 as it has at T42 for low elevation areas of the Arctic and Europe. While $\mathrm{q}$ is the LSWM variable, it is sensitive to hydrostatic changes due to differences in the topography used by CAM 3 and ERA-40 models. The SLP bias thereby de-emphasizes the topography and makes the primary features of interest over the oceans and lower-elevation lands more easily seen (Fig. 1j, k). However, at the LSWM resolution, the differences between $\mathrm{q}$ and SLP are pretty small (Fig. 2j, k). The NAST location error (low SLP over northern Europe, higher SLP over the Barents Sea), the missing Beaufort high (negative values of $q$ bias north of Alaska), and the generally lower SLP over the entire Arctic region (north of $60 \mathrm{~N}$, Greenland excluded) are all present at R12. Other broader features of particular interest, such as the dipole (cold bias north of warm over the longitudes 0-30W, with warm bias above in the stratosphere), the midlevel warm bias near 90E, the warm bias near the surface with cold bias aloft over the Beaufort Sea are adequately captured at R12 (Fig. 2c, f, i).

Very large values of the bias due to the mismatch in topography (between CAM3 and ERA-40) occur at small scales that are not present at the lower resolution. This is exemplified by the divergence bias near Greenland and the Himalayas. The divergence bias in Fig. 1 is calculated from the winds and is not as accurate as the vorticity biases also shown in that figure. Accordingly, several procedures were tested to calculate the divergence bias used by the LSWM. The divergence bias shown in Fig. 2 is calculated 
by a combination of Ekman pumping (lowest layers) and from geostrophic winds that are defined using a variable Coriolis parameter. The divergence calculated directly from the ERA-40 and CAM3 data was excessively large and judged to be unreliable, hence the derived divergence shown in Fig. 2. The reader should note that using either definition of divergence did not alter noticeably the basic structure of the forcing of the other variables, except for some influence upon the vorticity forcing at low levels (e.g. $\sigma>0.7$ )

The bias shown in Fig. 2 leads, via (1), to the forcing $\mathrm{F}$ shown in Fig. 3. Interpreting the forcing in relation to the bias is not as simple as negative temperature forcing where the bias is cool, for example. The relation between the forcing and the bias is complicated due to the linear operator. However, it helps to consider the dominant role of zonal advection at some levels. For example, the NAST in CAM3 is directed into western Europe instead of further north. This causes a negative SLP bias, and hence a positive upper troposphere vorticity bias peak, centered near $50 \mathrm{~N}, 15 \mathrm{~W}$. (see Fig. 2k, g, d, respectively.) Hence, the vorticity gradient is positive on the upstream side of the peak vorticity value there and the gradient is negative on the downstream side. The resultant forcing, if it were only due to zonal advection by a westerly wind, would be an east-west oriented dipole with the positive value upstream and the negative pole over western Europe. Such a dipole is seen in Fig. 3d, g. For the relative ridge in the SLP bias to the north (near $75 \mathrm{~N}, 5 \mathrm{E}$; Figs. $1 \mathrm{k}, 2 \mathrm{k}$ ) a dipole in the forcing would again occur, but the signs would be reversed. Hence a quadrapole pattern of forcing in the 'rectangular' region bounded by: $(60 \mathrm{~W}, 40 \mathrm{~N})$ and $(60 \mathrm{E}, 85 \mathrm{~N})$ can be expected from the zonal advection of the vorticity bias in the presence of westerlies. This pattern is found (Fig. 3d) in the middle and upper troposphere of the vorticity forcing and in the forcing of $q$ (Fig. 3j). Expressing the $q$ forcing in terms of SLP (Fig. 3k) changes the amplitude, but hardly changes the pattern. Similar to the vorticity forcing, zonal advection of the warm bias over western Europe and cold bias to the north explains the quadrapole pattern in the middle and lower troposphere of the temperature forcing (Fig. 3f) seen in that region. Finally, the bias and forcing of vorticity at low levels (Figs. 1g, 2g, 3g) can be expressed in terms of a stream function by inverting the La Placian operator and these are shown in Fig. 4g-i. The patterns for lowest level stream function bias and forcing are quite similar to (but smoother than) corresponding fields for $\mathrm{q}$ and SLP.

As described in GPT, the temperature field is linked with the sea level pressure field wherein cold temperatures are linked to higher SLP and warmer temperatures linked to lower SLP. The generally warmer temperature bias in the lower troposphere over most of the Arctic (Figs. 1f, i,
Fig. 2 Similar format as Fig. 1 except for the LSWM R12 horizontal and ten levels vertical resolution. Bias in vorticity (top row: a, d), divergence (middle row; b, e; divided by 10), and temperature (bottom row; c, f). Levels are shown for these multi-level fields are: $\sigma=0.05$ (left column) and $\sigma=0.55$ (right column). On the next page bias in vorticity (g), divergence (h; divided by 10$)$ and temperature (i) are shown at level $\sigma=0.95$ (left column). Also shown is the bias in $\mathrm{q}(=\ln \mathrm{Ps} ; \mathrm{j})$ and sea level pressure $(\mathbf{k})$ for this resolution

$2 \mathrm{f}, \mathrm{i})$ is thus associated with lower SLP over the region (Figs. 1k, 2k). As discussed in GPT, the relatively higher SLP bias (weakly positive) over the Barents Sea is due in part to the CAM3 NAST being far to the South. That is, there is higher SLP because fewer transient low pressure systems enter the Barents Sea in CAM3. Conversely, there is a strong negative SLP bias over the British Isles and across the Baltic due to that same NAST bias, since CAM3 sends too many frontal cyclones across that region. The fact that the Barents Sea positive max is much weaker than the Baltic Sea negative reflects the generally lower Arctic region SLP associated with the generally warmer low level temperatures. The LSWM analysis strengthens the association with temperature further. The patterns of near-surface temperature and $\mathrm{q}$ forcing (Fig. $3 \mathrm{i}, \mathrm{j}$ ) have a very similar pattern of extrema, as well as matching signs.

From geostrophic balance, one expects the near-surface vorticity and q (or similarly SLP) to be consistent, including their forcing. The LSWM shows such consistency. The near-surface forcing of vorticity and temperature (Fig. 3g, i) match the sign and pattern of the associated bias fields (Fig. 2g, i) in the LSWM. The resultant $q$ bias field (Fig. 2j) partly matches the q forcing (Fig. 3j) with additional contribution by the vorticity forcing (Fig. $3 \mathrm{~g}$ ). For example, CAM3's missing Beaufort high is a low pressure in the $\mathrm{q}$ bias centered north of Alaska across to eastern Siberia. The $\mathrm{q}$ forcing is negative north of eastern Siberia while the vorticity forcing at $\sigma=0.95$ (Fig. 3g) has peak positive forcing further east (north of Alaska). The combination of the two forcings matches the $q$ bias pattern with a peak just east of the peak in q forcing alone. Similarly, the Barents Sea anticyclonic bias is closely lined up with the $\mathrm{q}$ forcing, but there is strong anticylonic forcing of near-surface vorticity (Fig. 3g) nearby (a little to the east).

The subtropical jet near the northern west African coast is too weak in CAM3 while the north Atlantic subtropical jet extends too far south and east (i.e. it is too strong into western Europe). This may be deduced from the bias in stream function in the mid troposphere (Fig. 4d, e) deduced from the corresponding vorticity bias. The stream function near the northwest African coast is too high in the model while the stream function over northern Europe is too low. So the stream function meridional gradient is too large over much of Europe but too weak over northern Africa. Consequently, the upper tropospheric vorticity bias (Fig. 1d) 
a

$$
\text { At } \mathrm{Sig}=0.05
$$

VOR BIAS

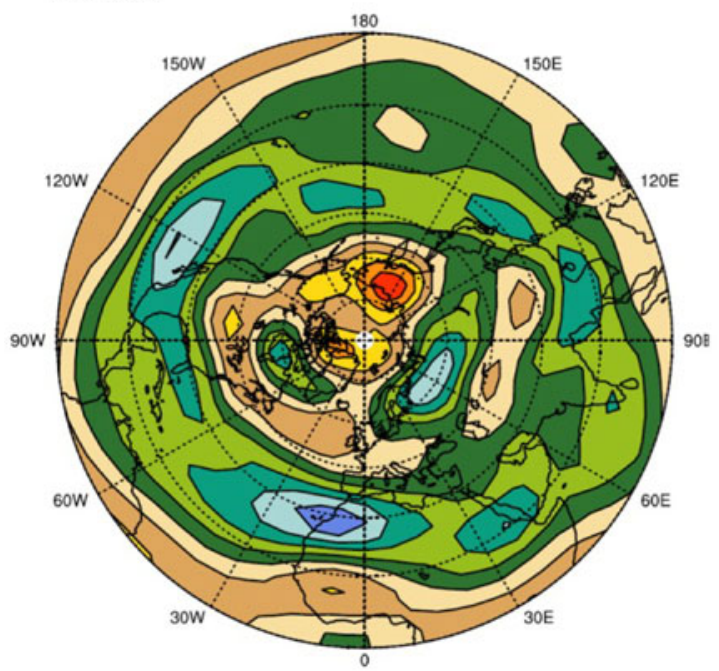

b DIV BIAS

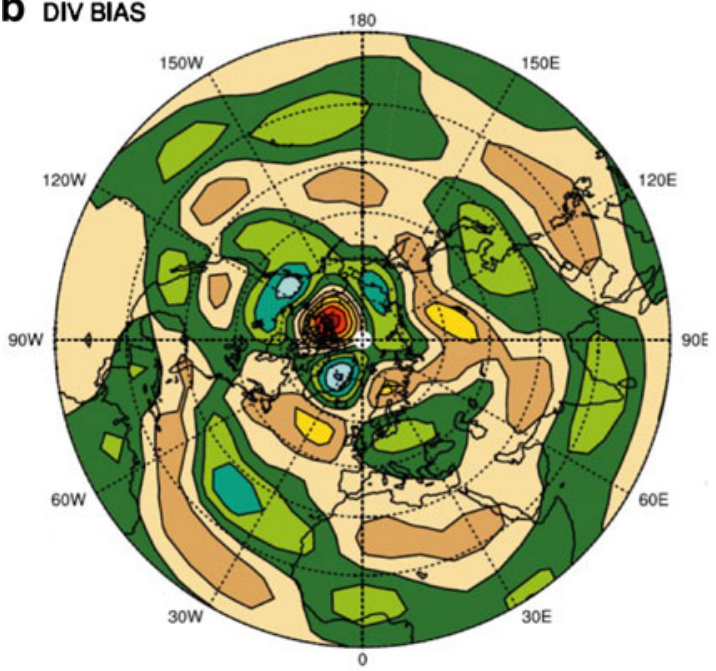

\section{TEMP BIAS}

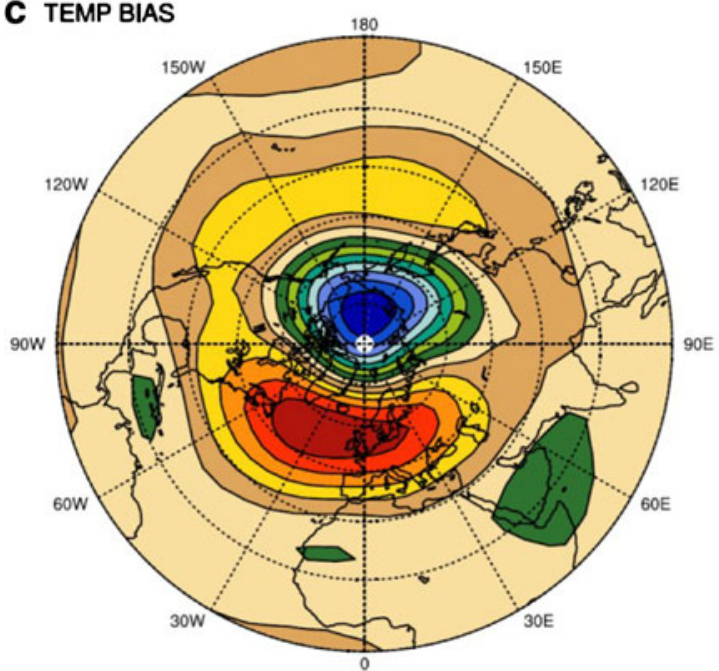

d

At $\mathrm{Sig}=0.55$

VOR BIAS
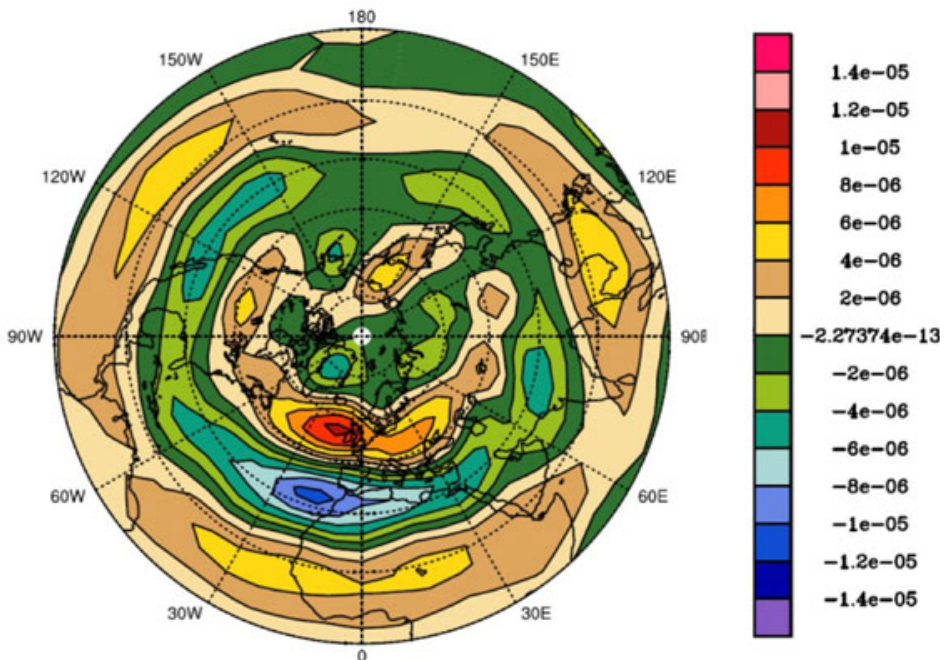

e DIV BIAS
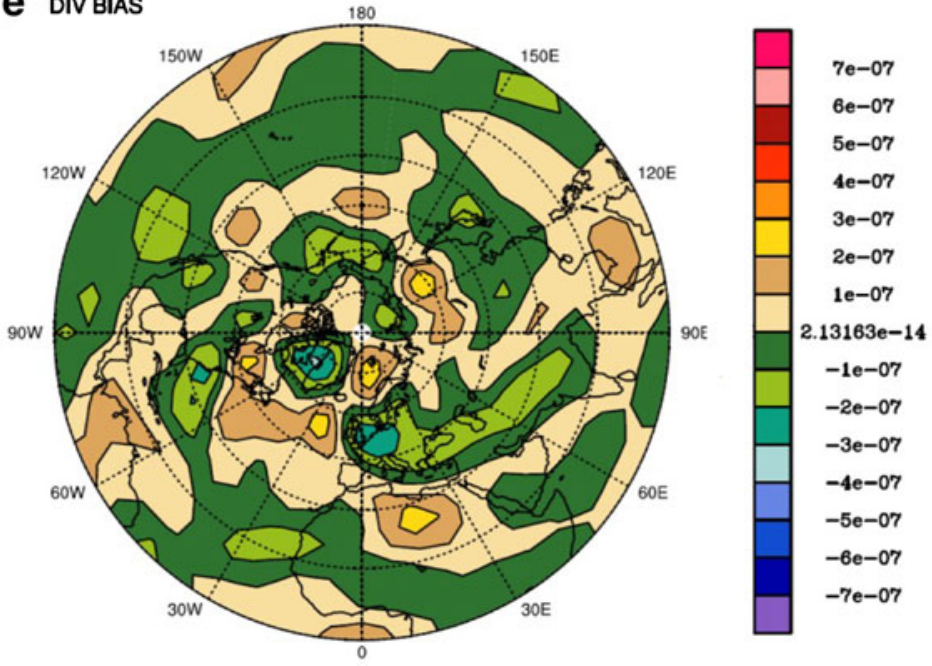

f TEMP BIAS

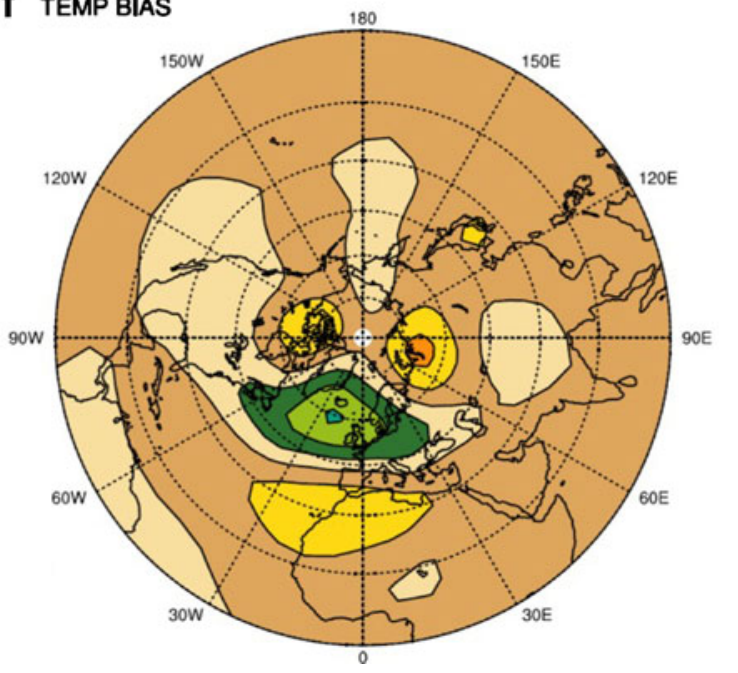




\section{g At Sig $=0.95$ \\ VOR BIAS}
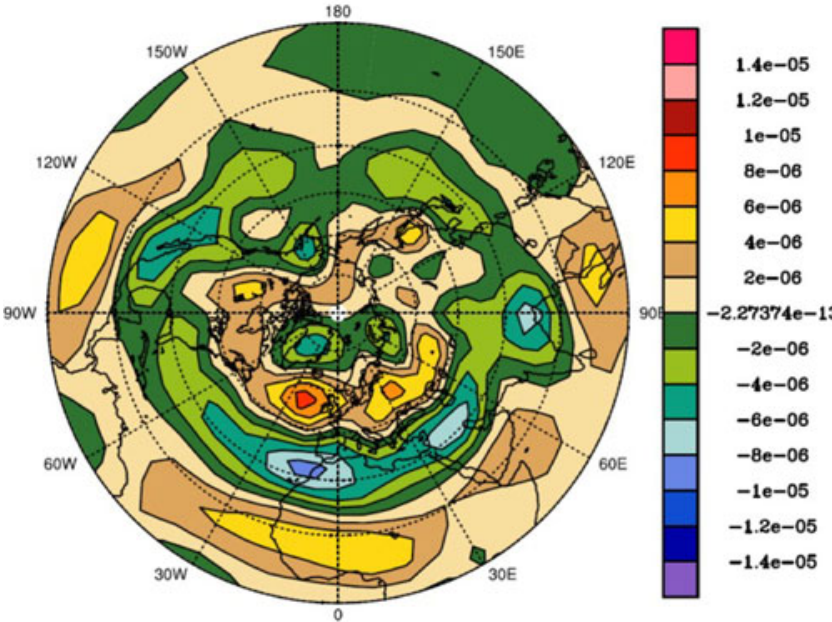

$\mathbf{h}_{\text {DIV BIAS }}$
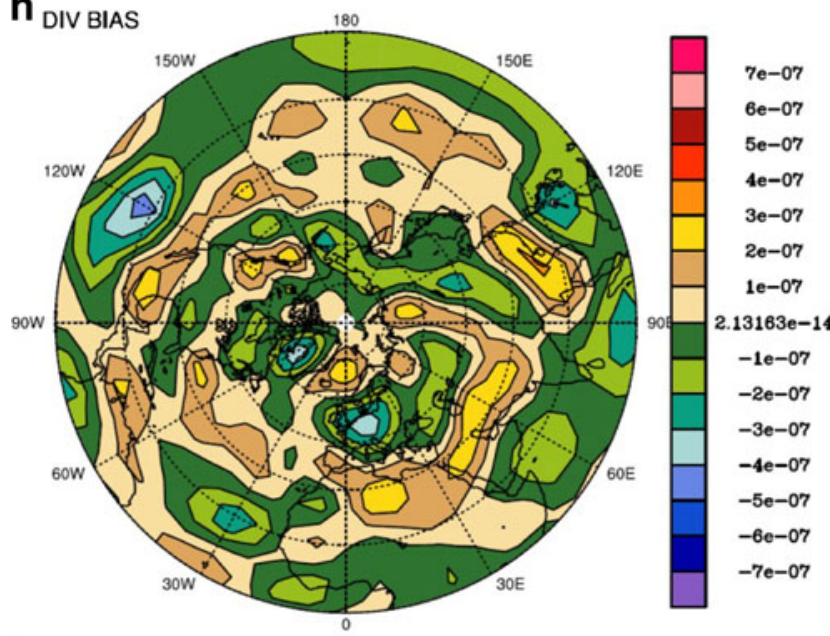

i TEMP BIAS
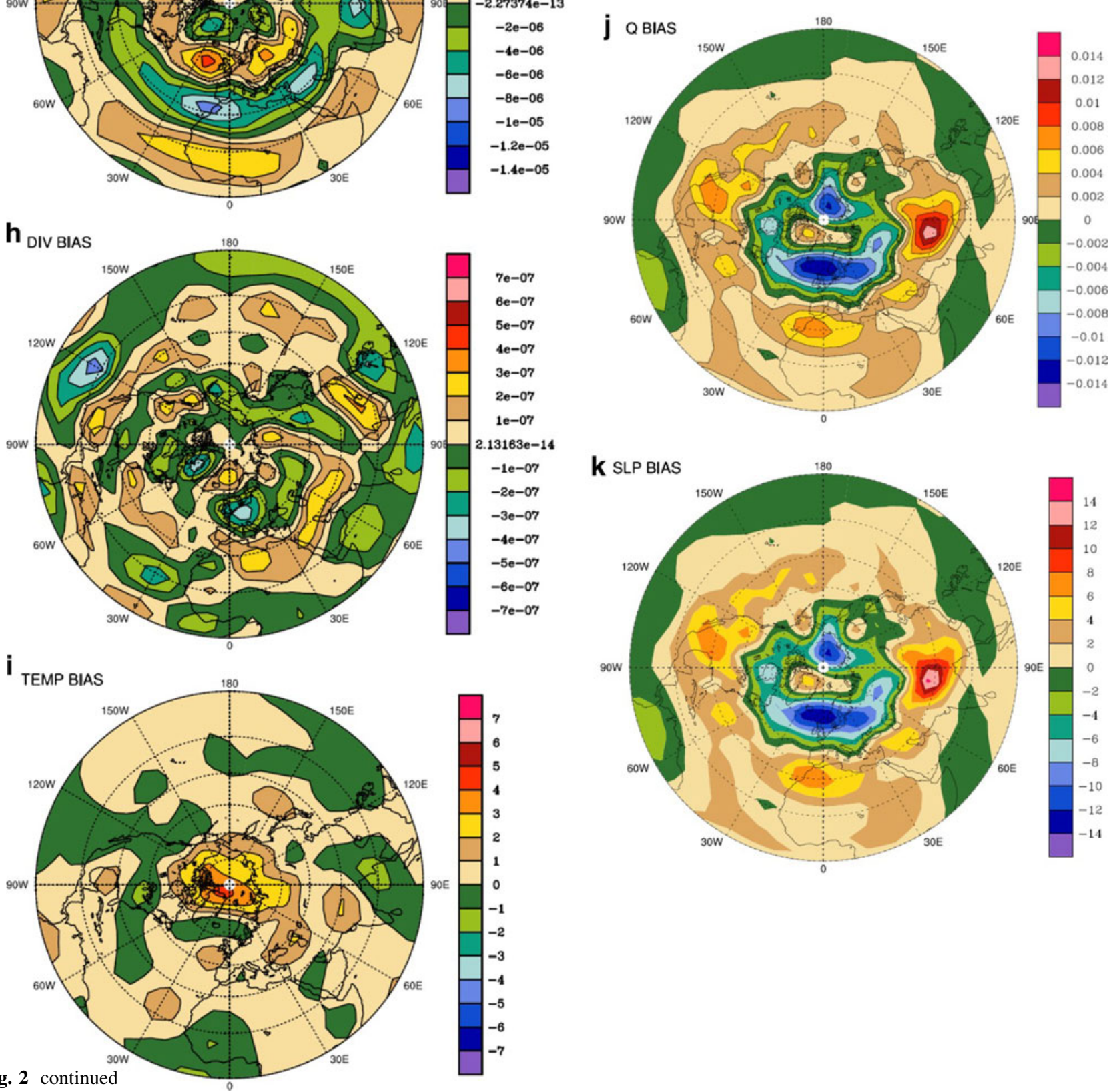

Fig. 2 continued 
a

VOR FORCING

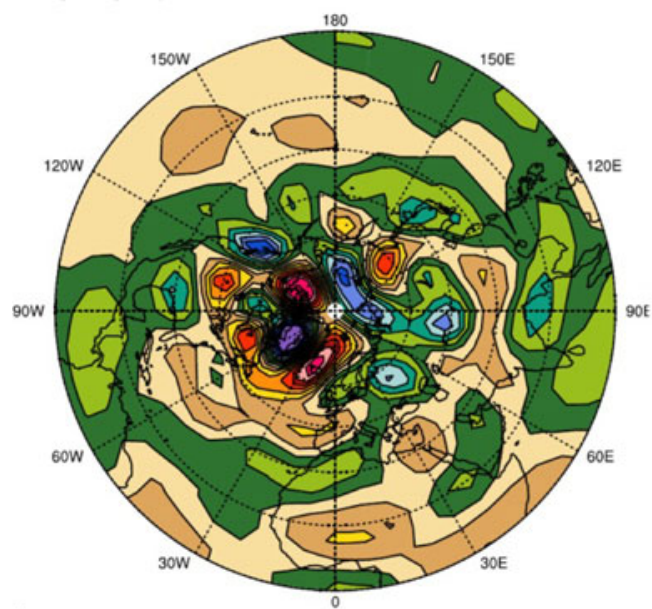

b

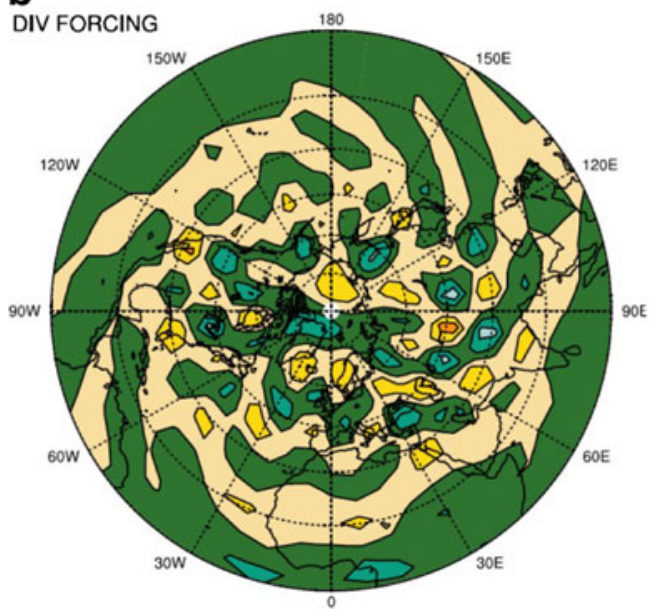

C

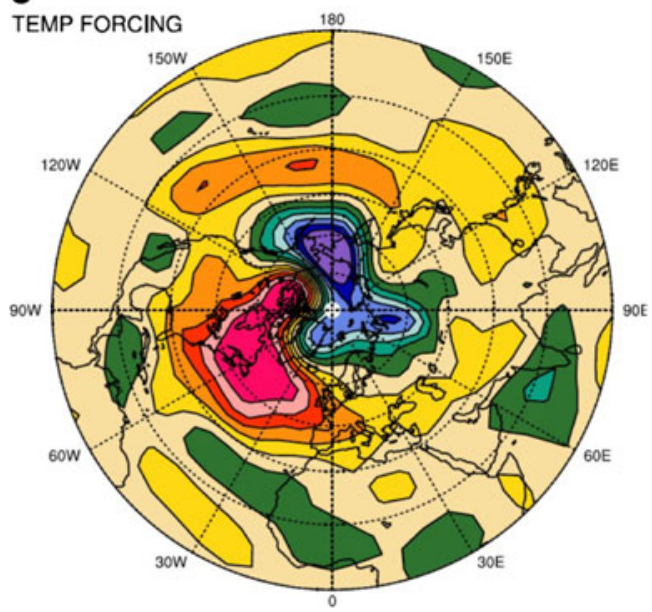

d

$$
\text { At } \operatorname{Sig}=0.55
$$

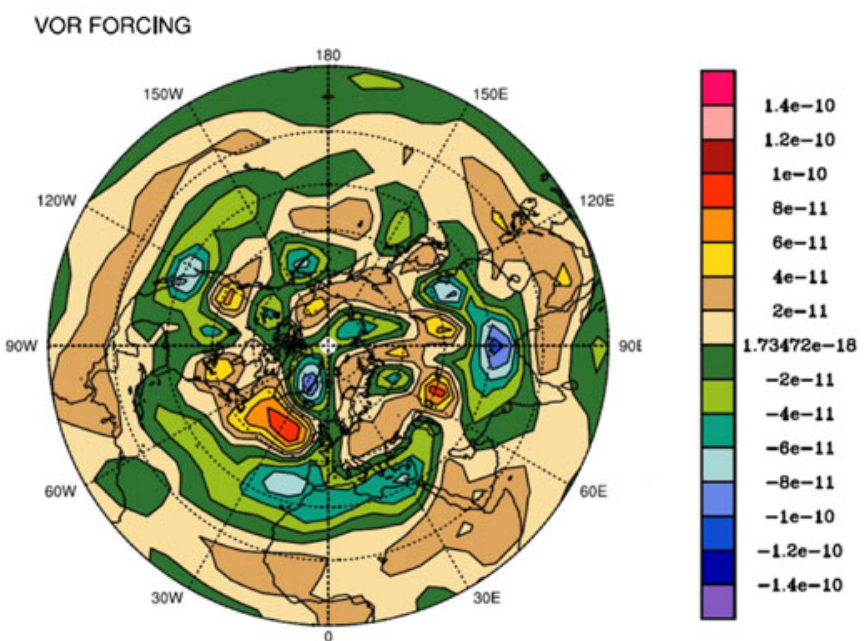

e

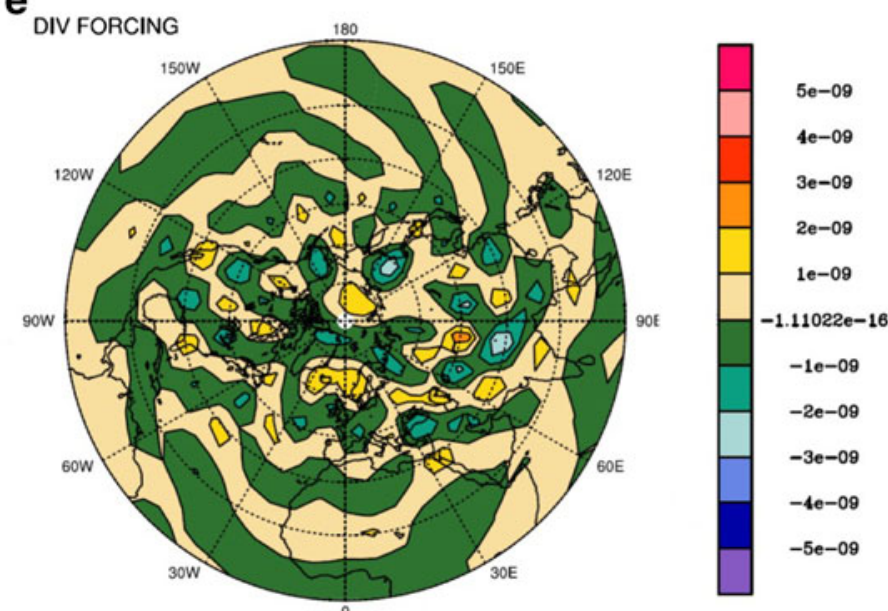

$\mathbf{f}$

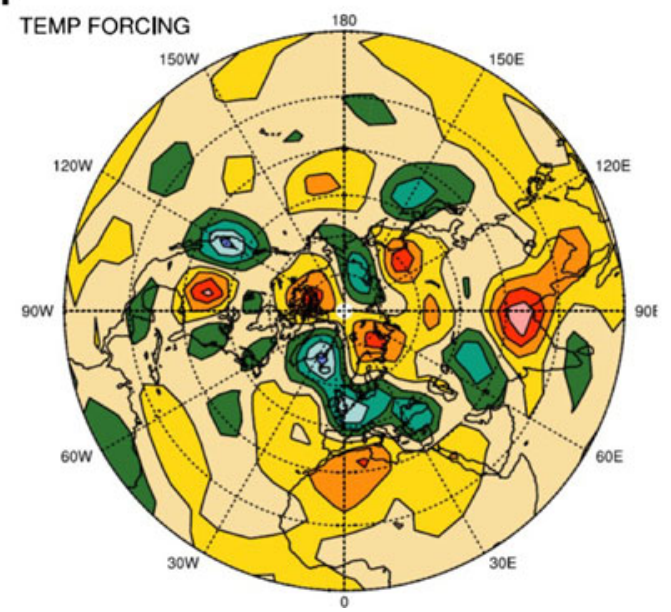

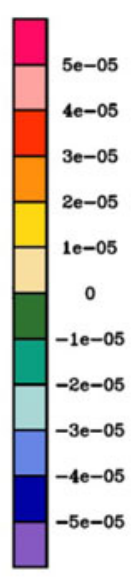

Fig. 3 Similar to Fig. 2 except for forcing fields calculated by the LSWM for R12L10 resolution

has a negative peak between those two jets and a positive maximum further south, at the west African coast (near $12 \mathrm{~N}$ ). These features are captured in the low resolution bias (Fig. 2d) and have corresponding extrema in vorticity forcing (Fig. 3d). The features in the forcing field may be more easily seen in Fig. 4f, which expresses the forcing in terms of the stream function. The middle tropospheric temperature bias (Fig. 2f) shows the enhanced meridional 
g At $\mathrm{Sig}=0.95$

VOR FORCING

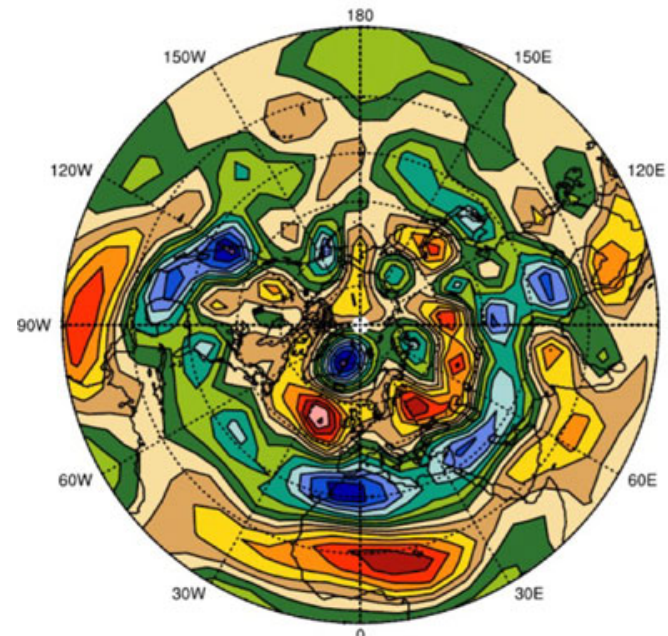

h

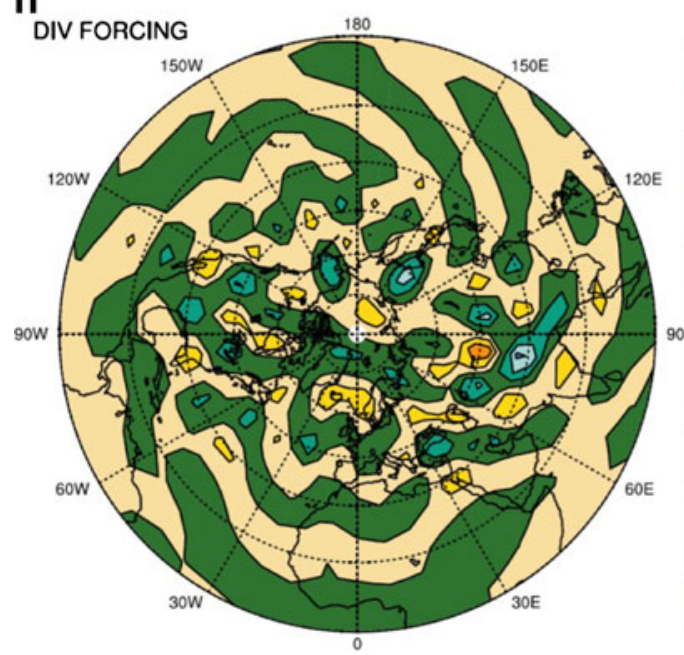

i

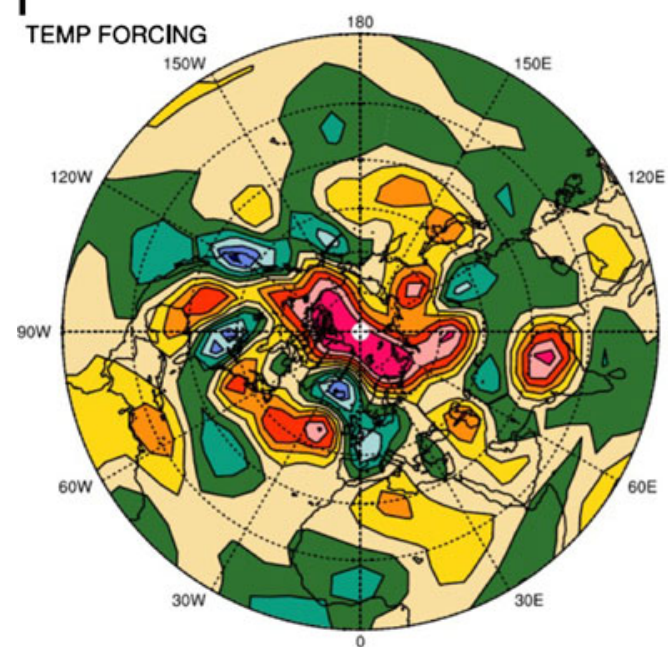

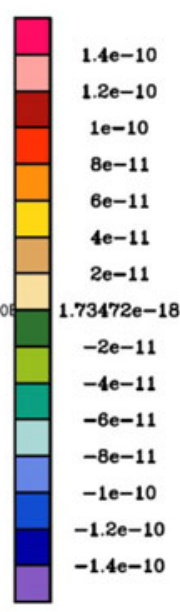
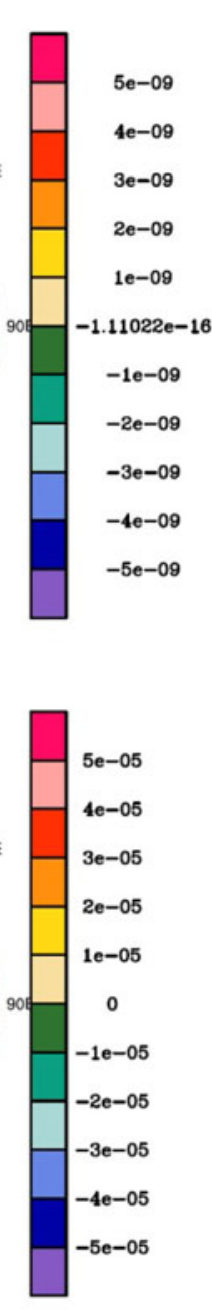

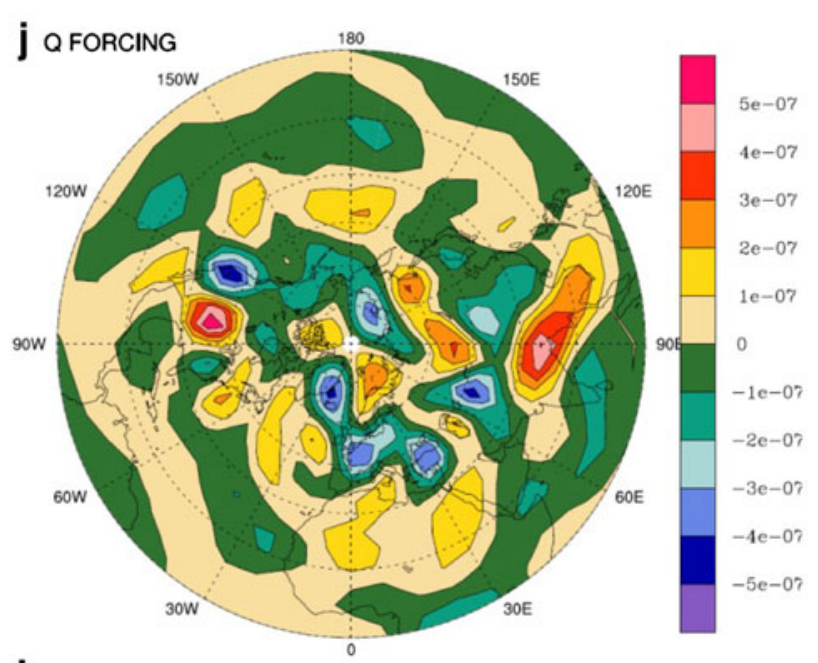

k

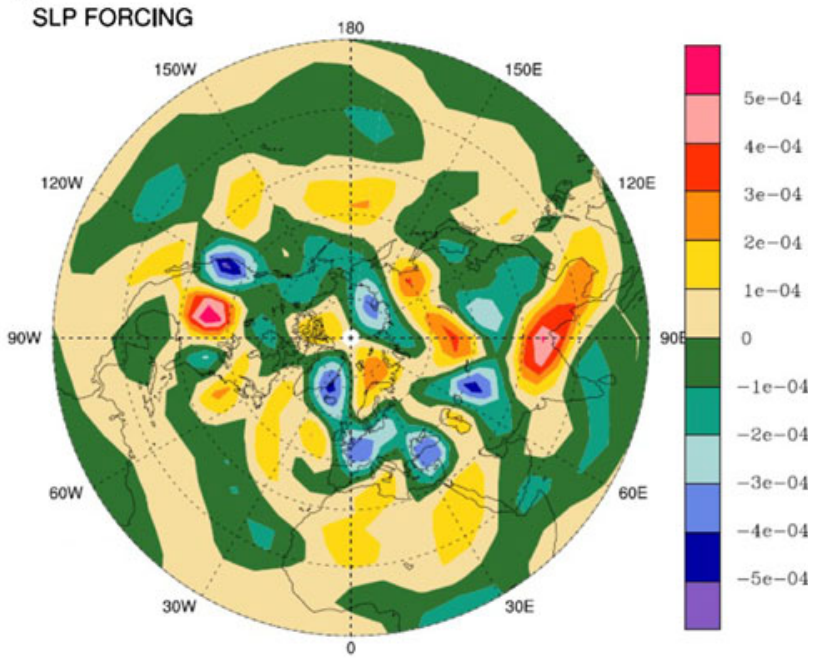

Fig. 3 continued 
a

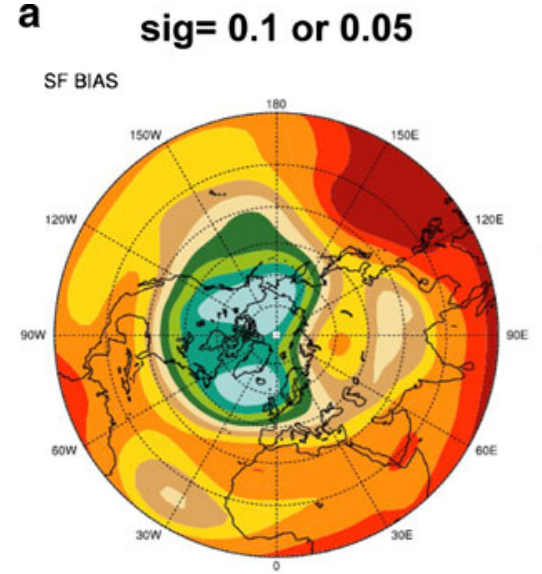

b $_{\text {SF BIAS }}$

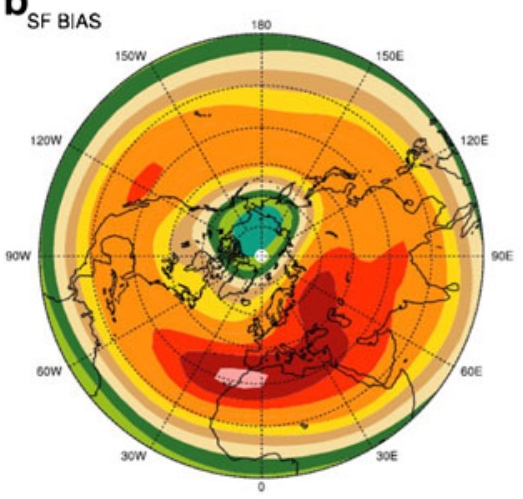

$\mathbf{C}_{\text {SF FORCING }}$

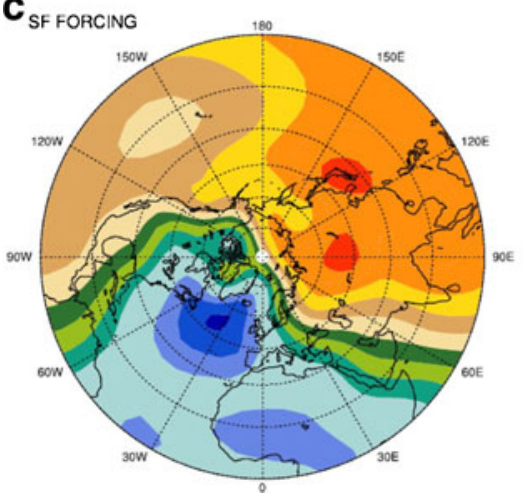

d

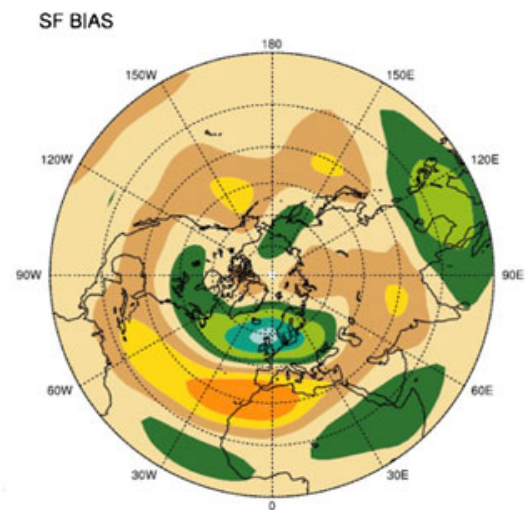

$\mathbf{e}_{\mathrm{SF} B \mathrm{AS}}$

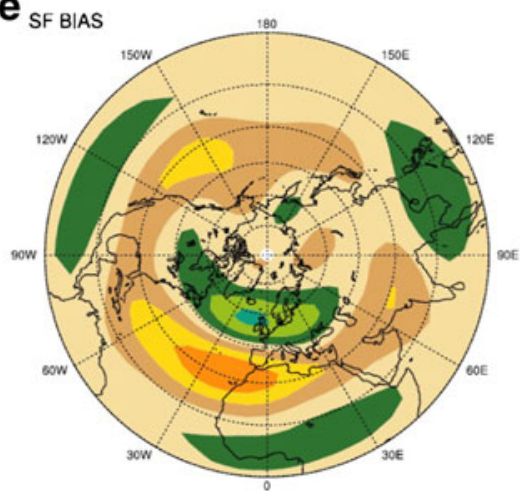

$f_{\text {SF FORCING }}$

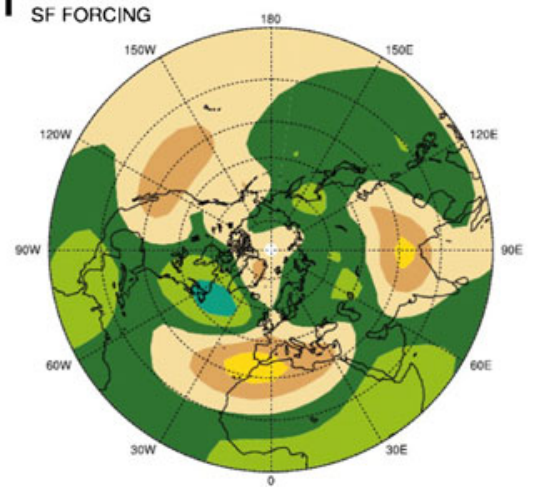

g

sig $=0.95$

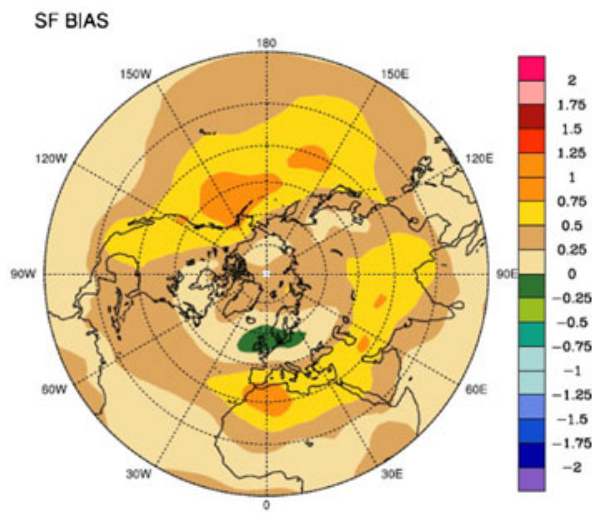

$\mathbf{h}_{\mathrm{SF} B \mid A S}$

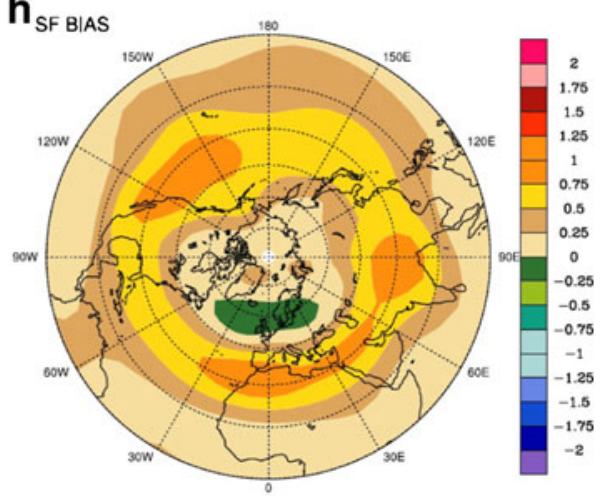

i SF FORGING

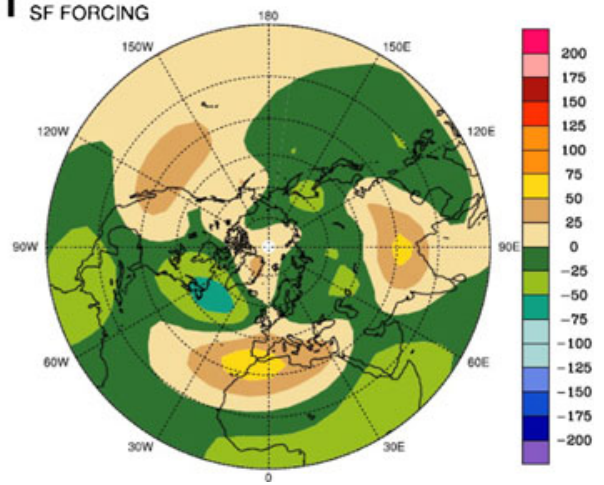

Fig. 4 Stream function based quantities produced by integrating corresponding vorticity-based quantities from Figs. 1, 2, 3. Top row stream function bias at levels $\sigma=0.1,0.5$, and 0.95 (left, middle, right panels) for T42 truncation. These may be compared with the corresponding vorticity biases shown in Fig. 1a, d, g. Middle row stream function bias at levels $\sigma=0.05,0.55$, and 0.95 (left, middle, right panels) for R12 truncation of the LSWM. These may be compared with the corresponding vorticity biases shown in Fig. 2a, d, g. Bottom row vorticity forcing of Fig. 3a, d, g expressed as a stream function tendency at levels $\sigma=0.05,0.55$, and 0.95 (left, middle, right panels) for R12 truncation of the LSWM temperature gradient near $45 \mathrm{~N}$ over Europe and adjacent Atlantic Ocean that one expects (from thermal wind balance) for this jet stream bias. The forcing is different from other places discussed in being dipolar in longitude at $45 \mathrm{~N}$ between longitudes $45 \mathrm{~W}$ to $45 \mathrm{E}$ (heating over the Atlantic, cooling over Europe). The European cooling extends into the Middle East and to the south is a maximum heating over Libya (and extending across north Africa); this dipole enhances the downstream end of that meridional gradient temperature bias.

The mid-tropospheric minimum temperature bias centered south of Iceland (near 60N, 20W; Figs. 1f, 2f) is also explainable from the forcing field. At upper and lower levels the forcing has a dipolar structure that partly straddles this temperature bias minimum, however the minimum in the bias is well within the area where a cooling forcing 


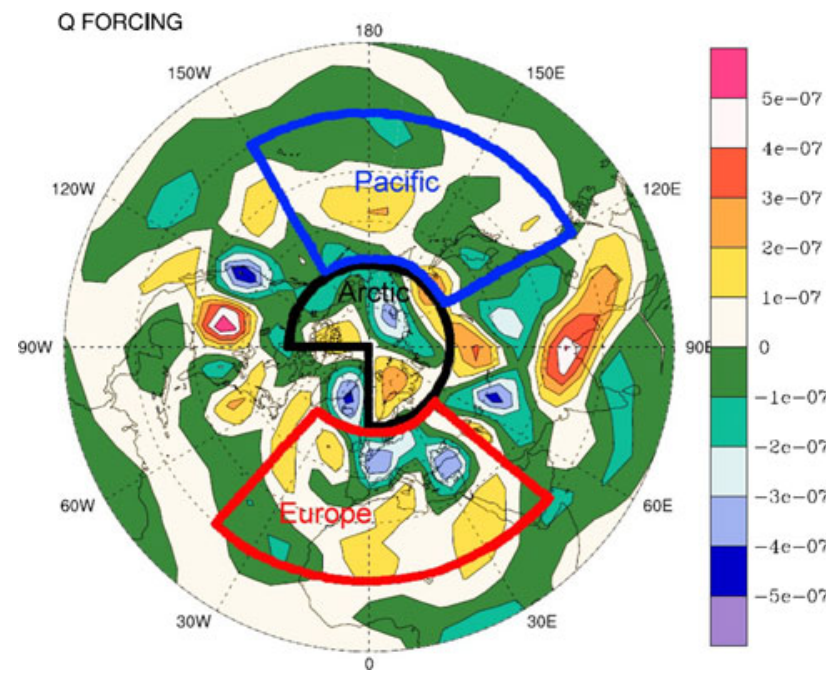

Fig. 5 'Arctic', 'Europe', and 'Pacific' sectors used when calculating the $\mathrm{q}$ fields shown in fig. 6 and tabulated in Table 1, superimposed on the $q$ forcing field shown in Fig. $3 j$

pole predominates (Fig. 3f). Qualitatively, the negative temperature bias is from the simulated NAST being too far south.

Near the west coast of continental Europe a lower tropospheric warm bias occurs. PGT noticed that the bandpassed transient heat fluxes (Fig. 2 in PGT) are larger in CAM3 than ERA-40 near this region (near 45N, 20W) and the diabatic heating bias (Figs. 1f, 31 in PGT; from excess precipitation in CAM3 along its NAST) is strongly positive in the same region. Hence both transient heat fluxes and excess diabatic heating contribute to the positive values in the temperature forcing and consequent warm bias off the west coast of Europe.

\section{LSWM solution fields in the Arctic}

The solution fields, by design, recover the input bias when the full global forcing is used. What is interesting is to examine the contributions of individual portions of the forcing to the SLP bias in two areas of specific interest: the Beaufort High and the Barents Sea region. Five forcing regions were defined based on capturing major features in the forcing pattern, while isolating the problematic Greenland region. (Greenland is not emphasized here because topography varies between the CAM3 and ERA40 models and $\sigma$ surfaces have large slopes near such large topographic features.) The regions are defined by these longitude-latitude ranges. 'Arctic' is from 0 to $270 \mathrm{E}$ and from $60 \mathrm{~N}$ to $90 \mathrm{~N}$. 'Europe' is from $40 \mathrm{~W}$ to $50 \mathrm{E}$ and from $15 \mathrm{~N}$ to $60 \mathrm{~N}$. 'Greenland' is from $270 \mathrm{E}$ to $360 \mathrm{E}$ and from $60 \mathrm{~N}$ to $90 \mathrm{~N}$. 'Pacific' is from $120 \mathrm{E}$ to $210 \mathrm{E}$ and from $15 \mathrm{~N}$
Fig. 6 LSWM solutions for $\mathrm{q}$ using the forcing, a over the whole globe-compare with Fig. 2j. Solutions for forcing only from individual sectors are shown in: b Arctic sector, c Europe sector, $\mathbf{d}$ Pacific sector, e Greenland sector, and $\mathbf{f}$ all remaining areas of the globe not included in b-e. Sectors b-d are plotted in Fig. 4. Clearly, most of the bias over the Arctic ocean is captured by the local forcing of the Arctic region

to $60 \mathrm{~N}$. All the other locations on the Earth not in those four regions are contained in the region labeled 'All Else'. Figure 5 shows the regions marked on the $q$ forcing shown in Fig. 3j.

For each of the five regions a bias solution is obtained. This discussion focuses on the solution for $\mathrm{q}$ bias in two regions: the missing Beaufort high and the relatively higher pressure over the Barents Sea and adjacent lands. The Beaufort high area of interest is bounded by $90 \mathrm{E}$ to $270 \mathrm{E}$ and $60 \mathrm{~N}$ to the pole. The other area of interest is labeled the 'European Arctic' and is bounded by $0-90 \mathrm{E}$ and $60 \mathrm{~N}$ to the pole. The $q$ bias solutions, one for each of the five regions, along with the total $\mathrm{q}$ bias are reproduced in Fig. 6. It is immediately clear that most of the $q$ bias in the two regions of interest is picked up by the local forcing of the Arctic sector (Fig. 6b). Visually, the Pacific sector forcing creates the positive q bias over eastern Siberia and over Alaska. The Pacific sector forcing also adds some positive $q$ bias to the European Arctic where the total bias (Fig. 6a) has a relative maximum (weakly positive) in the Barents Sea. The Europe sector forcing contributes to the general negative $\mathrm{q}$ bias over most of the Arctic region. The positive $\mathrm{q}$ bias over northern Greenland is well stimulated by the local forcing. Though not of prime interest here, it is noted that the Europe sector captures the bias locally including the negative $\mathrm{q}$ bias from the NAST location error and the positive $\mathrm{q}$ bias over northern Africa (related to the subtropical jet errors).

The $\mathrm{q}$ bias solution for each region of forcing is projected onto the $q$ bias over each area of interest, normalized by the total bias squared over the interest region, and multiplied by 100 to obtain the percentage of the total $\mathrm{q}$ bias that can be created by the portion of the forcing field in that region. Table 1 summarizes the contributions by the forcing in the five regions to each of the two areas of interest. It is immediately clear that the local (Arctic sector) forcing explains the bulk of the $\mathrm{q}$ bias in both areas of interest. This result is consistent with the visual inspection of Fig. 6 and with the discussion in GPT. For the European Arctic there is a sizable secondary contribution from the Greenland region forcing as well. The Greenland contribution is notable because the relevant dipolar structure in the forcing (Fig. 3j) includes a pole with much of its magnitude centered over Greenland. The Europe sector forcing has a sizable tertiary contribution to the European Arctic q bias, though with opposite sign; that result is 

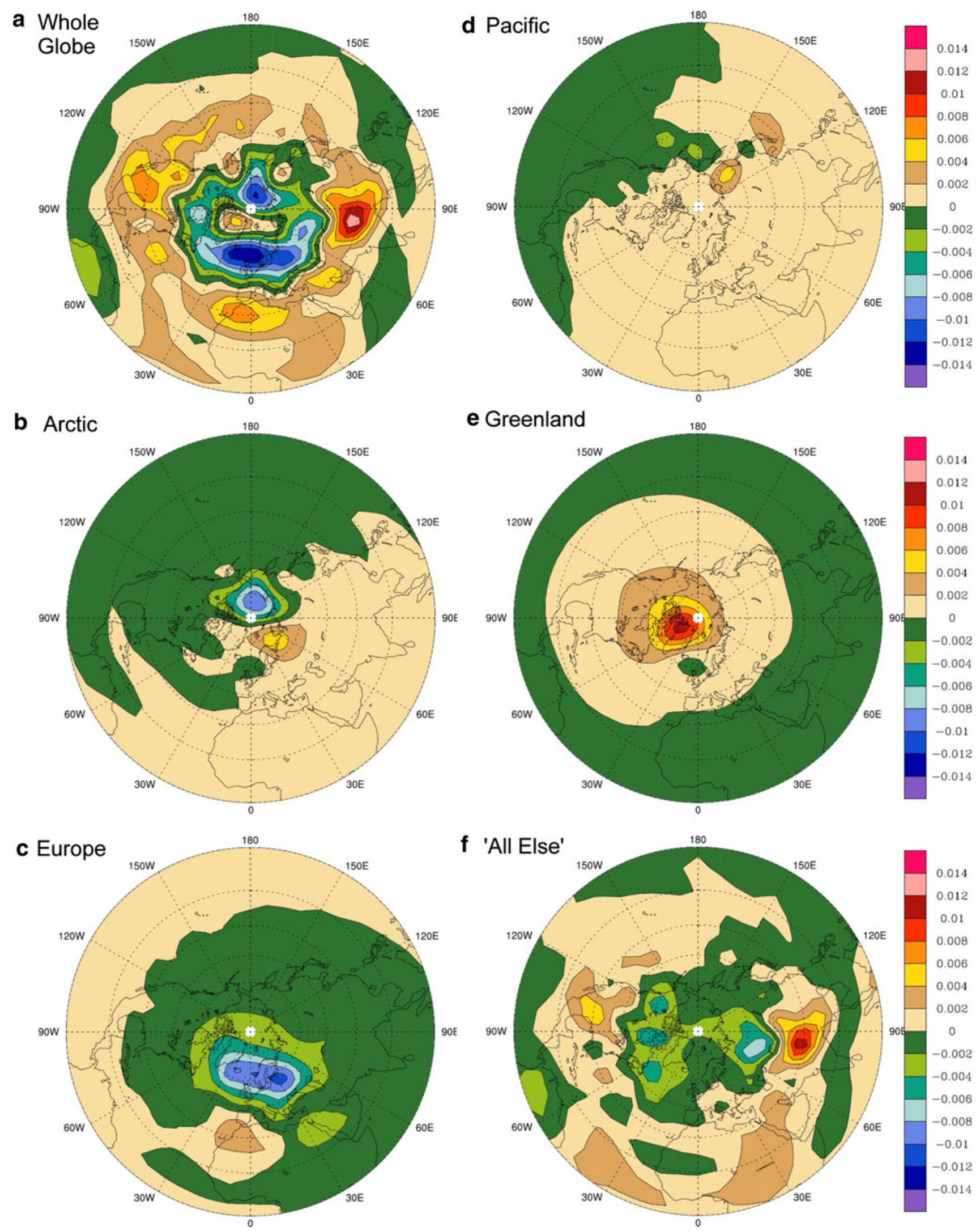
Table 1 Percent contribution to the $\mathrm{q}$ bias in two regions of the Arctic from the forcing in various areas

\begin{tabular}{lll}
\hline Forcing region & $\begin{array}{l}\text { European Arctic } \\
\text { (ridge in bias) } \\
60 \mathrm{~N}-90 \mathrm{~N} \\
0-90 \mathrm{E}\end{array}$ & $\begin{array}{l}\text { Beaufort high } \\
\text { (trough in bias) } \\
60 \mathrm{~N}-90 \mathrm{~N} \\
90 \mathrm{E}-270 \mathrm{E}\end{array}$ \\
\hline Arctic & 70.5 & 105.3 \\
$60 \mathrm{~N}-90 \mathrm{~N}$ & & \\
$0-270 \mathrm{E}$ & & 30.6 \\
Europe & -24.1 & \\
$15 \mathrm{~N}-60 \mathrm{~N}$ & & -45.2 \\
$40 \mathrm{~W}-50 \mathrm{E}$ & & \\
Greenland & 55.2 & -17.3 \\
$60 \mathrm{~N}-90 \mathrm{~N}$ & & \\
$270 \mathrm{E}-360 \mathrm{E}$ & & 26.6 \\
Pacific & 10.1 & \\
$15 \mathrm{~N}-60 \mathrm{~N}$ & & \\
$120 \mathrm{E}-210 \mathrm{E}$ & & \\
All else (everywhere & -11.8 & \\
else on the globe) & & \\
\hline
\end{tabular}

consistent with our earlier interpretation of the forcing from advection having a dipolar structure and the rather arbitrary nature of the boundaries for the Europe region largely excludes the forcing pole to the north and east of the forcing poles along the CAM3 NAST. The Pacific region does not have much contribution to the Beaufort high bias based on this analysis.

\section{Conclusions}

This study employed a linear stationary wave model (LSWM) to identify atmospheric forcing of the CAM3 model bias by transient and diabatic processes. Only the Northern Hemisphere in winter was shown. The study focused upon the lower tropospheric pressure bias over the Arctic region, though forcing and bias at other levels and regions of the Northern Hemisphere were also shown. While the model biases are richly detailed, the main features are captured by the LSWM which, of necessity, must perform its calculations at much lower resolution. Triangular truncation (T42) and 29 level CAM3 simulations were regridded to the Rhomboidal truncation (R12) and 10 levels of the LSWM.

Forcing and bias in the Arctic were emphasized, and focused upon two specific biases in the natural logarithm of surface pressure $(=q)$ field. Classical synoptics (Petterssen 1956) postulates a linkage between cold tropospheric temperature and high sea level pressure (SLP) and vice versa. Generally, over the Arctic the model bias is warm and the SLP bias is negative, consistent with classical theory. The forcing for the Beaufort high and the weak relative positive $\mathrm{q}$ in the Barents Sea are more complicated than that classical theory.

The forcing for the $\mathrm{q}$ bias can be related to the pattern of the bias of q. CAM3 has little or no discernable Beaufort High, resulting in a strong negative q bias over the Arctic Ocean centered near the dateline. The corresponding forcing for $\mathrm{q}$ is a bit to the west, with a negative anomaly near Siberia's Arctic coast at 150E. Near-surface (positive) vorticity forcing extends further east (covering much of the Arctic north of Alaska) so together the vorticity and $q$ forcing support the strongly negative SLP bias from 90E to $250 \mathrm{E}$ across the Arctic. The second primary bias of interest is a relative maximum in q near Novaya Zemlya within the generally negative $q$ throughout much of the Arctic. The forcing in that area is positive for $\mathrm{q}$ and negative for nearsurface vorticity (Fig. 3j, g, respectively) near Novaya Zemlya and roughly corresponds to the location of the positive $\mathrm{q}$ bias.

The contributions by different portions of the forcing to the $\mathrm{q}$ bias in the Beaufort high area and to the Barents Sea area are calculated by the LSWM. The local forcing is found to dominate. Other regions have contributions that could be related to extrema in the forcing field that could be paired with local Arctic bias extrema; such dipolar combinations were anticipated from horizontal advection contributions to the forcing. While the bulk of the forcing needed to create the two Arctic biases is local, as pointed out by GPT, a 'local' bias could be associated with the storm tracks (located some distance away) when the track error causes storms to affect (or not) the region of interest. GPT emphasized how the Barents Sea positive SLP bias was associated with vorticity equation terms activated by frontal cyclones that were not traveling as near the region in CAM3 as in ERA-40 data. To a lesser extent the Beaufort High bias was partly caused by the same frontal cyclone activated vorticity terms being too strong in CAM3. So, the picture that emerges from this study as well as GPT and PGT suggests improving the CAM3 bias in the Arctic as follows. Emphasis should be placed upon improving the local diabatic processes and upon the simulation of the frontal cyclone storm track properties, especially those for the NAST.

Acknowledgments This work was supported by NSF grant ATM 0354545. ECMWF ERA-40 data used in this study/project were provided by ECMWF from the main data server. The authors thank Dr. Grant Branstator and Mr. Andy Mai for providing the LSWM and for their assistance in setting it up for our study. The authors thank the members of NCAR's Climate Modeling Section, Computer Software and Engineering Group, and Scientific Computing Division for their contributions to the development of CAM3. Comments by two anonymous reviewers improved the manuscript. 
Open Access This article is distributed under the terms of the Creative Commons Attribution Noncommercial License which permits any noncommercial use, distribution, and reproduction in any medium, provided the original author(s) and source are credited.

\section{References}

Branstator G (1990) Low-frequency patterns induced by stationary waves. J Atmos Sci 47:629-648. doi:10.1175/1520-0469(1990) 047<0629:LFPIBS > 2.0.CO;2

Collins WD, Rasch PJ, Boville BA, Hack JJ, McCaa JR, Williamson DL, Kiehl JT, Briegleb B, Bitz C, Lin S-J, Zhang M, Dai Y (2004) Description of the NCAR community atmosphere model (CAM3). Technical report NCAR/TN-464 STR, National Center for Atmospheric Research, Boulder, CO, 226 pp

DeWeaver E, Bitz CM (2006) Atmospheric circulation and its effect on Arctic sea ice in CCSM3 simulations at medium and high resolution. J Clim 19:2415-2432

Grotjahn R, Pan L-L, Tribbia J (2011) Sources of CAM3 vorticity bias during northern winter from diagnostic study of the vorticity equation. Clim Dyn. doi:10.1007/s00382-011-0998-0
Pan L-L, Jin F, Watanabe M (2006) Dynamics of synoptic eddy and low-frequency flow (SELF) interaction. Part III: baroclinic model results. J Atmos Sci 63:1709-1725

Pan L-L, Grotjahn R, Tribbia J (2009) Sources of CAM3 temperature bias during northern winter from diagnostic study of the temperature bias equation. Clim Dyn 35:1411-1427. doi: 10.1007/s00382-009-0608-6

Petterssen S (1956) Weather analysis and forecasting, 2nd edn. McGraw-Hill, New York

Uppala SM, Kållberg PW, Simmons AJ, Andrae U, da Costa Bechtold V, Fiorino M, Gibson JK, Haseler J, Hernandez A, Kelly GA, Li X, Onogi K, Saarinen S, Sokka N, Allan RP, Andersson E, Arpe $\mathrm{K}$, Balmaseda MA, Beljaars ACM, van de Berg L, Bidlot J, Bormann N, Caires S, Chevallier F, Dethof A, Dragosavac M, Fisher M, Fuentes M, Hagemann S, Hólm E, Hoskins BJ, Isaksen L, Janssen PAEM, Jenne R, McNally AP, Mahfouf JF, Morcrette JJ, Rayner NA, Saunders RW, Simon P, Sterl A, Trenberth KE, Untch A, Vasiljevic D, Viterbo P, Woollen J (2005) The ERA-40 re-analysis. Quart J R Meteorol Soc 131:2961-3012 